Commentary/Humphreys \& Forde: Hierarchies, similarity, and interactivity in object recognition

\title{
Category-specific deficits: Insights from semantic dementia and Alzheimer's disease
}

\author{
Matthew A. Lambon Ralph ${ }^{\mathrm{a}}$ and Peter Garrard ${ }^{\mathrm{b}}$ \\ aDepartment of Experimental Psychology, University of Bristol, Bristol BS8 \\ $1 T N$, United Kingdom; ${ }^{\mathrm{b}}$ The Institute of Cognitive Neuroscience, Alexandra \\ House, London WC1N $3 A R$, United Kingdom. \\ matt.lambon-ralph@bristol.ac.uk p.garrard@ucl.ac.uk \\ www.psychology.psy.bris.ac.uk.psybris www.icn.ucl.ac.uk/
}

Abstract: Recent investigations and theorising about category-specific deficits have begun to focus upon patients with progressive brain disease such as semantic dementia and Alzheimer's disease. In this commentary we briefly review what insights have been gained from studying patients of this type. We concentrate on four specific issues: the sensory/functional distinction, correlation between features, neuroanatomical considerations, and confounding factors.

Although many reports of patients with category-specific disorders have been based on stable brain-damaged patients (e.g., CVA: Sacchett \& Humphreys 1992; HSVE: Warrington \& Shallice 1984), it is only relatively recently that data from patients with progressive brain disease have been used to inform this issue. This is despite the fact that semantic impairments in progressive disorders such as Alzheimer's disease (AD) and semantic dementia (SD) were established many years ago (e.g., Warrington 1975). We summarise below some of the insights that have been gained from this source of data (for a fuller account, see Lambon Ralph et al. 1998b).

The sensory/functional distinction. Although there have been a number of reported associations between category-specific impairment for living things and relatively poor sensory knowledge (e.g., Gainotti \& Silveri 1996), evidence for the complementary association (between nonliving things and functional knowledge) is sparse. One example was provided by a longitudinal analysis of definition and naming data of patients with $\mathrm{AD}$ (Lambon Ralph et al. 1997). Although the AD patients did not demonstrate category-specific differences in overall performance, Lambon Ralph et al. (1997) were able to show that a decline in the ability to name living things was associated with loss of sensory information whilst 
poorer naming of artefacts paralleled degraded functional knowledge. As noted by Humphreys and Forde $(H \& F)$, there is an increasing number of single cases that call the causality of this association into question. There are patients with category-specific deficits for living things with equivalent sensory and functional knowledge. Patients with semantic dementia, in contrast, seem to show the opposite combination - although conceptual knowledge is degraded overall, their ability to give or confirm sensory attributes is particularly affected (Lambon Ralph et al. 1998b 1999). If one controls for concept familiarity, there is little evidence that this pattern leads to the predicted category-specific impairment (Bozeat et al. 2000; Garrard et al., in press a; Lambon Ralph et al. 1998a; 1998b; 1999).

With this issue in mind, we have recently completed analyses of a feature database collected from intact control subjects (Garrard et al. 2001). Despite the fact that Shallice and Warrington's (1984) original proposal was based upon assumptions about the nature of those features that distinguished individual concepts, neuropsychological assessments of feature knowledge have not differentiated between shared and distinctive attributes. In the feature database, we found no difference in the number of distinctive sensory features listed for living and nonliving concepts. The number of distinctive functional attributes was, as predicted, greater for artefacts. Further analyses also revealed another category difference that may prove to be critical. The greater visual overlap/similarity of living things noted by $\mathrm{H} \& \mathrm{~F}$, extends to conceptual representation themselves. We (see also McRae \& Cree, in press) found that shared features were much more prominent for living than nonliving concepts.

Correlated features. Explanations of category-specific deficits that focus upon the importance of intercorrelated features predict that the direction of the category-difference should be related to severity (though the two best known positions make opposite predictions: Gonnerman et al. 1997; Tyler et al. 2000). Two recent studies including a relatively large number of AD patients found no positive evidence for either version of the theory (Garrard et al. 1998; in press c). The first, a cross-sectional analysis, found that the direction of the category-specific difference was not related to severity. Rather specific deficits for living or nonliving concepts were both more likely to be found in the most severely affected patients. More recently, after adding longitudinal data to the previous cross-sectional study, we found no positive evidence for the predicted cross-over in category-differences when plotted as a function of severity (either in terms of overall disease progression or the degree of semantic impairment). The lack of an effect in the target patient group (AD) may be explained by further analyses of the feature database, noted above. First, the number of significantly correlated feature pairs as a proportion of the total number of possible features pairs is extremely small, suggesting that any effect of feature co-occurrence is likely to be weak. Second, intercorrelation is confounded by feature distinctiveness - it is shared features that tend to correlate with others, again suggesting that it may be the distribution of shared versus distinctive features, which is critical.

Neuroanatomical considerations. Although it did not support the correlated feature accounts of category-specificity, the crosssection AD study (Garrard et al. 1998) did find positive evidence in favour of neuroanatomical influences. Those cases with relatively poor artefact knowledge/naming had more AD pathology in parietal regions, supporting the predominant view that there is a temporal versus frontoparietal difference at the heart of categoryspecific deficits. The semantic dementia cases are, again, a puzzle in this regard. The atrophy in these cases (e.g., Mummery et al. 2000 ) is focused upon the anterolateral aspects of the temporal lobes bilaterally and includes the inferior temporal gyrus. One might expect this to lead to relatively poor performance for living things as is found in some patients with HSVE for whom the distribution of pathology is somewhat similar (though it tends to involve medial as well as lateral temporal regions bilaterally: Gainotti et al. 1995).
Confounding factors. We finish where H\&F began. Concept familiarity strongly influences the accuracy of patients with semantic impairment (Bozeat et al. 2000; Lambon Ralph et al. 1998a) and it certainly explains some, but not necessary all, of the underlying difference in category-specific cases (e.g., Funnell \& De Mornay Davies 1996). We would argue that familiarity is no more a "nuisance" or confounding factor than different sensoryfunctional weightings of concepts - there is plenty of evidence that familiarity should be regarded as a critical part of understanding this issue. The magnitude of category-differences is reduced dramatically once familiarity controlled stimuli are used for assessment and there are at least three demonstrations of an interaction between category-specific differences and familiarity (Funnell \& De Mornay Davies 1996; Gainotti \& Silveri 1996; Lambon Ralph et al. 1998b). Implemented computational models of conceptual knowledge show that when familiarity is included during training, not only does it influence the models' overt performance (Lambon Ralph et al., in press), but also the nature of the derived semantic representations is changed. Familiar concepts tend to take up greater amounts of the semantic "space," making them much less vulnerable to simulated damage (Rogers \& McClelland, submitted). 